\section{Graphorrhea as a 'Soft' Bipolar Sign}

\section{Ahmed Naguy ${ }^{1 *}$ and Haya Al-Mutairi ${ }^{2}$}

${ }^{1}$ Child and Adolescent Psychiatrist Centre for Mental Health (KCMH), Kuwait ${ }^{2}$ Director General, $\mathrm{KCMH}$, and Consultant Psychiatrist, Adan Hospital, Kuwait

\section{Keywords: Bipolar spectrum disorder; Graphorrhea}

\section{Short Communication}

Juvenile bipolar disorder (JBD) poses a clinical conundrum, both diagnostically and therapeutically. In part, this could be ascribed to the developmentally-insensitive diagnostic criteria of our current classificatory systems, fairly common atypicality of presentations in this population, mixed psychotic ultra-rapid cycling course, high rates of comorbidities, and overall less-than-optimal response to medications [1]. Bipolar Spectrum Disorder (BSD), or Cade's disease, stipulates a history of a depressive episode with 'red flags' that draw the clinicians' attention to the possibility of underlying bipolarity for what clinically manifests as 'pseudounipolar' depression [2]. These are depicted in Table 1. And conceivably, this is of paramount importance with prognostic and therapeutic implications as antidepressants use in such cases could destabilize mood, induce manic shifts and/or accelerate cyclicity [3]. Hypergraphia, or graphorrhea, has been tied to organicity, e.g. interictal personality and stroke but also reported in schizophrenia and mania, where the patient has a compulsive tendency to write at a length. Mungas defined hypergraphia as a tendency to excessive writing that goes beyond any social, occupational, or educational requirements. This needs to be differentiated from organic automatic writing behaviour where writing perseveration without elaboration is evident [4-7].

Here, we are reporting a case of 'pseudounipolar' depression in an adolescent with a striking graphorrhea that turned out to be in BSD after antidepressant exposure. We opine that graphorrhea in context of mood disorders to be considered the visual analogue of logorrhea, and hence, a 'soft' bipolar sign.

A 17 year-old, Jordanian, Female, youngster was casualty petitioned by her parents for 3 week history of insomnia, anorexia, ostensibly low and dampened mood, anergia, aprosexia, being indrawn, and notably hypergraphia, unusual for her, all reflecting self-derogatory, depressive cognitions coupled with passive death wishes. No obvious current psychosocial stressors. No genetic load. No past psychiatric encounter. For safety concerns, she was admitted to inpatient service. Baseline laboratory investigations, including TSH, toxic screen, EEG and neuroimaging were all negative. HAM-D scored profound depression.

MSE revealed a fair, ruddy, chubby youngster that looks her stated age, downcasted eyes, slouched, psychomotor sluggish, soft-

- Recurrent depression, possibly seasonal

- Brief depression (less than 3 months)

- Early-occurring first-onset depression (before the age of 25)

- A first-degree relative with bipolar disorder

- Hyperthymic predisposition

- Atypical depression

- Severe psychotic depression

- Postpartum onset

- Antidepressant-emergent mania or hypomania (bipolar III)

- Poop-out of antidepressants acutely (tachyphylaxis)

- Failure of three or more antidepressants trials

Table 1: Soft Signs of Bipolarity

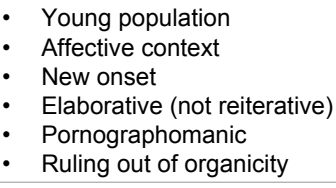

Table 2: Graphorrhea as a soft bipolar sign

spoken with terse, skimpy answers only to direct succinct questioning, restricted affectivity with reported hollowed mood, depressive themes and passive death wishes. No psychotic features could be detected.

Fluoxetine was started at $10 \mathrm{mg}$ am pc, with PRN alprazolam $0.25 \mathrm{mg}$. Over 2 days, mood very soon brightened and she looked activated. Few days later, she began to be verbose, hyperactive, overly familiar, disinhibited, with fitful sleep, inappropriate jocularity, and continued graphorrhea that extended to graffiti . SSRI-induced mania was entertained and fluoxetine was discontinued and patient was kept under close observation with PRN lorazepam IM. Over next week, she escalated to psychotic mania, now endorsing grandeur and persecutory delusions and mood turned mercurial with dysphoric quality instead. Olanzapine $10 \mathrm{mg}$ was instituted, up titrated to $20 \mathrm{mg}$ with tangible improvement over 2 week duration. She was discharged and followed up at week 4,8 and 12 with a plateau of euthymia, sound sleep, treatment adherence, and above all no more graphorrhea. She is now back to school, faring well, only put on some weight that was addressed by a dietitian.

As this case amply portrays, graphorrhea, retrospectively, was the only sign that could have pointed to bipolarity lurking in the background. As similar cases abound in the literature, we opine that clinicians should be vigilant as well as cognizant interpreting graphorrhea in mood setting as a visual analogue of logorrhea, indicative of accelerated thought processes, and hence, a 'soft' sign of bipolarity. Kraeplin noted that "manics may produce astonishing number of documents all from the pleasure in writing". We posit some criteria for graphorrhea as a soft bipolar sign summarized in Table $2[8,9]$.

\section{Disclosures}

Authors declare no conflicts of interest nor financial affiliations or industrysponsored research.

\section{References}

1. Geoffroy PA, Jardi R, Etain B (2014) Bipolar disorder in children and adolescents: a difficult diagnosis. Presse Med 43: 912-20.

2. Ghaemi SN, Ko JY, Goodwin FK (2002) Cade's disease and beyond:

*Corresponding author: Ahmed Naguy, CAP Unit, KCMH, State of Kuwait, Tel: 96565541937; E-mail: ahmednaguy@hotmail.co.uk

Received March 30, 2015; Accepted April 27, 2015; Published May 03, 2015

Citation: Naguy A, Al-Mutairi H (2015) Graphorrhea as a 'Soft' Bipolar Sign. J Psychiatry 18: 293 doi: 10.4172/2378-5756.1000293

Copyright: @ 2015 Naguy A, et al. This is an open-access article distributed under the terms of the Creative Commons Attribution License, which permits unrestricted use, distribution, and reproduction in any medium, provided the original author and source are credited 
misdiagnosis, antidepressant use and proposed definition for bipolar spectrum disorder. Can J Psychiatry 47: 125-34

3. Ghaemi SN, Hsu DJ, Soldani F (2003) Antidepressant use in bipolar disorder: the case for caution. Review article. Bipolar Disorders 5: 421-433.

4. Kalamangalam GP (2009) Hypergraphia in temporal lobe epilepsy. Ann Indian Acad Neurol 12: 193-4

5. Benbadis SR (2006) Hypergraphia and the diagnosis of psychogenic attacks. Neurology 67: 904.
6. Hermann BP, Whitman S, Arnston P (1983) Hypergraphia in epilepsy: is there a specificity to temporal lobe epilepsy? J Neurol Neurosurg Psychiatry 46: 848-853.

7. Van Vugt $P$, Paquier $P$, Kees $L(1996)$ increased writing activity in neurological conditions: a review and clinical study. J Neurol Neurosurg Psychiatry 61: 51-514.

8. Emeagwali N, Bailey R, Azim F (2012) Textmania: text messaging during the manic phase of bipolar I disorder. J Health Care Poor Underserved 23: 519-522.

9. Mondimore FM (2005) Kraeplin and manic-depressive insanity: a historical perspective. Int Rev Psychiatry 17: 49-52. 